\title{
Process Research in the Ivory Tower
}

\author{
Nathaniel S. Finney*
}

\begin{abstract}
Despite widespread perception to the contrary, training in process chemistry research can be incorporated seamlessly into the academic environment. The challenges and benefits associated with establishing a process research laboratory at the University of Zurich are discussed, and this program is presented as a successful model for expanding the educational mission of the University to include training in process chemistry.
\end{abstract}

Keywords: Educational mission · Process chemistry · Training

Is the Ivory Tower an appropriate venue for process chemistry research? The preceding articles provide a vibrant overview of the state of the art in process chemistry, and paint a picture of it as a pursuit brimming with new discoveries and developments; however, a point worthy of note is that, with two exceptions, the articles were contributed entirely by industrial researchers. Were this issue dedicated to, e.g., recent developments in $\mathrm{C}-\mathrm{C}$ cross-coupling methods (certainly an area of great industrial importance), a much more even balance of academic and industrial contributions might be expected. Two correlated observations and an important conclusion can be drawn: that process chemistry research is an exciting aspect of molecular science albeit conducted almost exclusively in industry; that despite its importance and central innovative character, process research is sorely neglected by academia; and, finally, that academic training in process chemistry is an unmet need at the academic/industry frontier [1].

Despite perception to the contrary, training in process chemistry logically fulfills part of an academic chemistry de-

${ }^{*}$ Correspondence: PD Dr. N.S. Finney

Organisch-chemisches Institut

Universität Zürich-Irchel

Winterthurerstrasse 190

$\mathrm{CH}-8057$ Zürich

Tel.: +41446354283

E-Mail: finney@oci.unizh.ch partment's educational mission. It is difficult to conceive of the discipline of organic chemistry without an integrated industrial component, any more than one could conceive of the discipline of economics without an economy. While it is certainly not the case that training chemists for industry is the sole function of a University chemistry program, the fact remains that the majority of organic chemists pursue industrial careers. As such, part of a complete educational program should include the training necessary to excel as a process chemist.

If process chemistry is so central to an industrially-relevant field of endeavor and yet process training is so conspicuous in its absence, how can such training best be incorporated into an academic chemistry program? How can academic process chemistry be best conceived to enrich the combined community of academic and industrial chemists? The Organic Chemistry Institute (OCI) at the University of Zurich (UZH) has created a laboratory for process research (Labor für Prozessforschung, LPF) to address just these questions; however, the lack of precedent for process research training in academia posed numerous challenges in establishing the LPF. Of particular concern were two issues: who should be trained, and how should the program be integrated into the existing University environment?

An important starting assumption was that complete, rigorous training in process chemistry should involve conducting research in a relevant context, and that this would be best facilitated by working with one or more corporate partners to be sure to focus on 'real life' problems. Such an academic/industry joint venture was anticipated to provide a training environment that would accurately reflect the time and cost pressures, the GMP regulatory constraints, and the intellectual property (IP) and technological transfer issues invariably associated with industrial process chemistry.

Issues of IP, confidentiality and regulatory constraints impinge upon some definitions of academic freedom and raise the question of who would be appropriate to train in such a program. It was clear that trainees should already possess a strong organic chemistry background, i.e. beyond one of ordinary skill in the art. In addition, they should be in a position where working on proprietary research which could not be published or presented (at least prior to protection of the intellectual property) would nonetheless be a net positive for their future career.

Integrating the program into the existing UZH environment required addressing several concerns. It would be essential to ensure the program and its participants would be fully engaged in the dynamic intellectual environment of the OCI. In recreating a corporate research context, it would clearly be important to avoid creating a separate "culture-within-a-culture'. The program would also need to be established in a way that would take full advantage of the existing world-class OCI infrastructure. An important and related issue would be ensuring the availability of the specialized infrastructure associated with GMP analytics and kiloscale production, with as little burden as possible to the University as a whole.

While there are doubtless many ways to overcome these challenges, in establishing the LPF they were addressed as follows 
[2]. It was decided that the primary trainees would be postdoctoral researchers, because the requirements for confidentiality and IP protection precluded trainees expecting to write an openly published thesis. Researchers with PhDs would clearly meet the requirement for a strong pre-existing background in organic chemistry. Properly handled, the constraints on open publication/presentation would not be a problem for the co-worker in training, nor for the University because the entry of the broadly trained chemist into the open employment market constitutes an unconventional albeit important aspect of academic productivity.

It was further decided that a 2-year postdoctoral term would be sufficient for a rigorous initial training in process chemistry. A consequence of this decision, which guaranteed frequent turnover of the primary researchers, was that it was necessary to establish a core group of permanent LPF employees. This technical staff, already expert in process chemistry, serves to train new postdocs, assures continuity of project management, and guarantees maintenance of the specialized skill sets associated with GMP regulation, analytics and production.

With the trainee pool identified and the associated internal components of the LPF defined, it remained to address the integration of the program into the existing UZH environment. A key component of the LPF is the 'leadership team', which necessarily includes the Heads of Research \& Development, Analytics, and Production [2]. However, the team also includes the Director of the LPF and a Chemical Advisor. Both of these positions are held by OCI faculty, and this is an important component of keeping the LPF fully engaged in the intellectual and educational activities of the OCI and the University as a whole.

By creating the LPF within the OCI, complete access to the remarkable infrastructure, from laboratory facilities to the full suite of routine analytics was assured. The specialized equipment required for GMP analytics has been handled entirely through the LPF's external budget. As such, there has been an additional benefit to establishing the LPF: the University gains access to analytics not otherwise available in a conventional modern organic chemistry institute. In a similar vein, instruments of interest to the OCI as a whole and the LPF in particular - a powder X-ray diffractometer with an automated sample changer and a reaction calorimeter - have recently been purchased jointly.

The need for specialized building infrastructure associated with kilo-scale GMP production - infrastructure rarely if ever found in academic laboratories - was fortuitously addressed long before the founding of the LPF, when the new OCI was be- ing built. During the 1970s, when the new UZH science campus was being designed, two-story cellulose-packed gravity chromatography columns were still in routine use for the purification of crude natural product isolates (a process often taking 2 3 months!). As a result, a special high-bay laboratory was included in the design of the new OCI. Ironically, between the design of the building and its final occupation, flash-column chromatography and large-scale HPLC had advanced to the point that the towering cellulose columns were obsolete, and the high-bay lab was never used for its original purpose. The space remained unoccupied for a number of years, until a former UZH PhD student, Dr. Dieter Beer, recognized it as suitable for the construction of a kilo lab for smallscale commercial production.

After founding a company, Carbogen, an arrangement was made for the company to lease the space from the OCI, as well as gain access to the OCI analytical infrastructure. After several years of incubation at UZH, Carbogen outgrew the available space and constructed its own facilities in Aarau, Switzerland. Following this departure, the LPF was formed as an independent research unit within the OCI by Prof. Hans-Jürgen Hansen, formerly the Head of Process Research at Roche, Basel, in collaboration with the first corporate partner, Cilag AG (Schaffhausen), whose process group was headed by Dr. Max Rey, a former UZH Oberassistant [3]. Since its inception, the LPF production facilities (capable of scale-up to $c a$. $10 \mathrm{~kg}$ ) have been upgraded and maintained through the LPF's external budget.

In this way, the UZH has established the LPF as a unique and much-needed academic training program in process chemistry. This integrated program at the academic/industry interface ensures that the incoming PhD trainees learn everything from the details of process chemistry to non-infringing route design, patent analysis and protection of IP, GMP analytics and production, and tech transfer - all under 'real world' constraints on time and costs. The LPF has operated successfully for more than 10 years, with a series of corporate partners, has placed several postdocs in industrial positions each year, and has a record of complete employment of former postdocs - all testaments to the success of this endeavor and the receptiveness of the chemical industry to academic process chemistry training.

In a broader context, the successful establishment of the LPF dovetails with the OCI's ongoing commitment to equip its undergraduate, graduate and postdoctoral researchers with the knowledge and skills they need to excel in their future careers.

At the undergraduate level, process chemistry naturally unifies several emerging themes in undergraduate instruction. As global awareness of the impact of human activity on our environment has grown, 'green' chemistry and industrial sustainability have become issues that are routinely addressed in introductory organic chemistry. However, it is difficult, at the blackboard or on the $<1 \mathrm{~g}$ scale of an undergraduate laboratory, for these ideas to take on any real meaning. Process chemistry provides a natural context for introducing students to the scale of industrial production and the essential 'systems analysis' inherent to the process chemist's mindset; it makes it clear that chemistry does not exist in a vacuum, but rather is coupled to sourcing, practical implementation, cost and time constraints, waste generation and long-term feasibility. This allows the undergraduates to move forward with open eyes into the 'real world' of chemistry. At the graduate level, the students benefit from direct interactions with the LPF postdocs and staff, which raises both their awareness of and interest in process chemistry. (Indeed, historically, some of the LPF's most successful postdocs have been recruited directly from the OCI graduate program.)

As further extensions of the spectrum of industry-oriented educational offerings within the OCI, the formal degree program in Business Chemistry (Wirtschaftschemie) and accredited apprenticeship training (Lehrlingsausbildung) deserve special mention. The former is a parallel degree program in which students complete the full requirements for a degree in chemistry while pursuing a parallel study of business and economics. Such a program prepares scientifically trained individuals for the task of leading technology-driven businesses. From project managers to CEOs, the business sector benefits from experts with such dual training. The apprenticeship program is a University activity with a direct parallel to training programs in industry. Most major chemical companies provide apprenticeship programs as a vehicle to recruit technical lab staff. The OCI at UZH and the Laboratory of Organic Chemistry (LOC) at ETH-Zurich provide structured apprenticeship training to young adults in search of alternative entry into technical career options. In a three-year mix of theoretical and practical experience, the apprentice is prepared to conduct chemical experiments on a professional level suitable for entrance into a major industry or academic research laboratory. This program develops the human resource infrastructure crucial to a healthy technology-based economy like that of Switzerland, Europe or the US.

Coming full circle, we can ask again, "Is the Ivory Tower an appropriate venue for process chemistry research?" The success 
of the LPF answers a resounding, "Yes!" Process chemistry training is not only appropriate in the Ivory Tower, it is arguably an essential part of a complete modern educational program in chemistry. It is hoped that the solutions that have worked for the LPF will serve as an inspiration to others to take on these challenges.

Received: August 8, 2006

[1] For recent comment on the absence of process chemistry training in academia, see: M.F. Lipton, A.G.M. Barrett, Chem. Rev. 2006, 106, 2582-2582.

[2] The internal structure of the LPF is one of the subjects addressed in a preceding article in this issue. See: T. Bader, Chimia 2006, 60, 534 .

[3] As an additional historical note, the bond between university activities in Zurich and industrial enterprise in Basel was forged long ago by patriarchs like Karrer at UZH and Ruzicka at the ETH. The tradition was carried forward and broadened to greater Switzerland under their reign and that of their successors. As such the parallel pursuit of pure academic research and academic/industrial synergy has been thriving for numerous decades. The modern embodiment of this relationship owes much of its success to the roots set down in the historical development of Zurich's twin premier academic chemistry research centers. 